\title{
La interculturalidad en la sociedad peruana y la formación del docente intercultural
}

DOI: $10.46932 /$ sfjdv2n2-129

Received in: March 1st, 2021

Accepted in: May 30th, 2021

\section{Dra. Yolanda Ramírez Villacorta \\ $\mathrm{PhD}$}

Docente Principal en la Facultad de Educación de la Universidad Nacional Mayor de San MarcosUNMSM

Domicilio: Jirón Francisco Bolognesi 695-D504. San Miguel. Lima-Perú

E-mail institucional: yramirezv@unmsm.edu.pe

\begin{abstract}
Peru is recognized as a pluricultural and multilingual country, with more than 50 original ethnic groups (Andean-Amazonian), to which we would add Afro-Peruvians and international migrants. However, the country vision has been homogenous, around the European target and the Spanish cultural heritage, hiding cultural diversity. The relations between cultures have been asymmetric, expressed in discrimination, marginalization, exclusion, on the basis dominant-dominated opposition; majority-minority. Teachers have been trained in this vision of the country and have been oriented to transmit information from the Western world and European knowledge, without assessing ancestral knowledge of cultural groups existing in the country. Currently, we seek to change that paradigm and has incorporated the proposal of the intercultural approach for relations between cultures and also for education. The classrooms are now multicultural. The new national educational policy marks an unavoidable challenge: to create a new curricular model to train intercultural teachers, reinforcing in them didactics and competences, capable of valuing and recovering knowledge of cultural diversity, to fulfill the role of educating in interculturality and forming citizens intercultural in a double dimension: to respond to the country and to act in the context of globalization.
\end{abstract}

Keywords: interculturality, cultural diversity, intercultural competences, teacher training, intercultural education.

\section{RESUMEN}

El Perú se reconoce como un país pluricultural y multilingüe, con más de 50 grupos étnicos originarios (andinos-amazónicos), a los que sumaríamos afroperuanos y migrantes internacionales. Sin embargo, la visión país ha sido homogénea, en torno al blanco europeo y la herencia cultural española, ocultando la diversidad cultural. Las relaciones entre culturas han sido asimétricas, expresadas en discriminación, marginación, exclusión, desde la oposición dominante-dominado; mayoría-minoría. Los docentes han sido formados en esa visión de país y han estado orientados a transmitir información del mundo occidental y conocimientos europeos, sin valorar saberes ancestrales de los grupos culturales existentes en el país. Actualmente, se busca cambiar ese paradigma y se ha incorporado la propuesta del enfoque de interculturalidad para las relaciones entre culturas y también para la educación. Las aulas son ahora pluriculturales. La nueva política nacional educativa marca un reto ineludible: crear un nuevo modelo curricular para formar docentes interculturales, reforzando en ellos didácticas y competencias, capaces de valorar y recuperar saberes de la diversidad cultural, para cumplir con el rol de educar en interculturalidad y formar ciudadanos interculturales en una doble dimensión: para responder al país y para actuar en el contexto de la globalización. 
Palabras clave: interculturalidad, diversidad cultural, competencias interculturales, formación docente, educación intercultural.

\section{A MODO DE INTRODUCCIÓN}

Como se sabe, toda sociedad genera cultura, la misma que podemos graficarla como la cobertura institucional para que funcione el sistema social y éste se estructure de manera que pueda sobrevivir a los cambios sociales, políticos, económicos, ideológicos y normativos, con la suficiente capacidad de adaptación y resiliencia para que los conflictos, que todo cambio provoca, sean superados y convertidos en eficientes procesos de renovación de la sociedad.

La sociedad se concretiza en la presencia de diferentes grupos sociales, empezando por la familia como grupo primario que hace posible el inicio de la socialización de los individuos, así como el aprendizaje de la cultura (endoculturación). A la familia se suman la escuela, el centro de trabajo, la parroquia, el vecindario, entre otros, como espacios que refuerzan la integración a la sociedad y el desarrollo de la identidad cultural, esto es: "me convierto en miembro de la sociedad y me identifico con sus normas, ideas y costumbres, las mismas que cumplo y defiendo” (Ramírez, 2002)

En las diferentes sociedades del mundo se reconoce la existencia de grupos sociales más grandes, cuyos individuos interactúan en base a lazos socio económicos y culturales, tanto o más complejos que los de la sociedad que los alberga y que no tienen fecha de expiración. Estos grupos se identifican como grupos étnicos, pueblos indígenas o pueblos originarios.

En el caso de la sociedad peruana existen aproximadamente 50 pueblos indígenas, en los andes y la amazonía, los mismos que tienen idiomas propios, lo que ha llevado a que, en la década de los '60 del siglo pasado, el antropólogo José María Arguedas sintetice al Perú como un "País de todas las sangres" (Arguedas, 1964). Actualmente, caracterizamos al Perú como un país multilingüe y pluricultural y hemos pasado de un paradigma oligárquico excluyente a uno incluyente, populista, aunque homogenizador, por lo que encontramos la presencia de la diversidad cultural en los diferentes espacios geográficos e institucionales de nuestra sociedad (C.I. Degrégori, 2003).

En el contexto descrito, destaca el panorama educativo actual constituido por aulas pluriculturales, conformadas con estudiantes de una procedencia diversa a nivel geográfico, étnico, lingüístico y cultural, que demanda de un profesorado con nuevas competencias, conocimientos, actitudes, didácticas y propuestas pedagógicas que le permitan el manejo educativo de ese tipo de aulas, además de considerar un nuevo tipo de relaciones interpersonales con la comunidad educativa del entorno. Sin embargo, la formación del docente para desempeñarse en contextos de multi y pluriculturalidad, con respuestas de 
interculturalidad, no ha considerado en sus planes curriculares los requerimientos específicos de la nueva educación intercultural para todos.

El papel del profesorado en esta nueva configuración de la escuela y la educación es absolutamente trascendental. Es más, el papel de los docentes como educadores y no como simples transmisores de información supone nuevas responsabilidades sociales, puesto que ahora se les exige fomentar en la escuela un espíritu tolerante, de respeto y convivencia, en el marco de los principios democráticos de igualdad de oportunidades y de solidaridad. Sin embargo, no se puede negar que aún hay muchos profesores que todavía permanecen pasivos, en medio de sus aulas tan diversas culturalmente, lo cual tiene significativas repercusiones en el funcionamiento de una escuela democrática, plural y heterogénea.

Es por ello que la presente comunicación busca aportar algunas reflexiones y propuestas en torno a lo que es la interculturalidad en el Perú, la educación intercultural y la formación de los docentes interculturales que respondan al reto de la interculturalidad en la escuela, para poder atender a todos los estudiantes en el reconocimiento de su legitimidad personal y cultural, aplicando en las actividades educativas los principios de cooperación, solidaridad y convivencia armónica en la diversidad cultural de nuestro país.

\section{EL CASO DE LA SOCIEDAD PERUANA: LA HERENCIA COLONIAL, LAS RELACIONES ENTRE CULTURAS Y LA EDUCACIÓN.}

Identificar, en el territorio peruano, los diversos modos de vida desarrollados por los diferentes grupos étnicos que han existido con anterioridad a la época Inca y española/europea, nos lleva a reconocer que esa diversidad ha generado diferentes formas y procesos de relacionamiento cultural. Sin embargo, en la historia del país, el proceso de la invasión y conquista española nos ha dejado una herencia colonial que nos hace sobrevalorar lo europeo en desmedro de lo indoamericano, que insiste en remarcarnos que los mestizos somos herederos de lo español/europeo, superiores a todo lo que represente lo indígena y no hispánico, que se traduce en relaciones interculturas asimétricas, con trato desigual, expresadas como marginación, exclusión, asimilación y definidas por la oposición de dominante - dominado, superiorinferior.

Es el principal trauma que hemos heredado de la conquista española: relaciones distorsionadas con las condiciones materiales de existencia, condiciones que están marcadas por la diversidad de ecosistemas y zonas de vida que hace de Perú el poseedor de 101 zonas de vida de las 112 identificadas por Holdridge, en el mundo. Esta herencia, negativa y deformada, se ha alojado en la conciencia e impide ver de cerca las relaciones reales y concretas de nosotros con nosotros mismos y con nuestro entorno natural y ambiental. 
La consecuencia evidente de este proceso de relacionamiento entre los diversos culturales fue el afianzamiento de la discriminación étnico/cultural. Nos hemos convertido en un pueblo "racista", pero por la "marca cultural", donde la segregación parte de nosotros mismos, por vergüenza de lo que somos: un pueblo marcado por su etnicidad y por la opresión sobre las comunidades andinas y amazónicas.

Las relaciones asimétricas, marcadas en las relaciones entre las diferentes culturas y sus correspondientes grupos sociales, han llevado a que, en la sociedad peruana, se limite la participación activa de los pobladores indígenas en la política (toma de decisiones) y en la educación (menos aprendizajes). Es significativa la discriminación totalmente racista que expresa Alejandro O. Deustua, quien llegó a ser Rector de la Universidad Nacional Mayor de San Marcos, en su libro "La Cultura Peruana" (1937):

\begin{abstract}
¿¿Qué influencia podrán tener sobre esos seres, que solo poseen la forma humana, las escuelas primarias más elementales?. ¿Para qué aprender a leer, escribir y contar, la geografía y la historia y tantas otras cosas, los que no saben vivir como personas, los que no han llegado a establecer una diferencia profunda con los animales, ni tener ese sentimiento de dignidad humana principio de toda cultura?"... "el indio vive sin interés alguno, bajo el imperio exclusivo de las necesidades materiales que satisface como las bestias, que son sus únicos modelos, y peor que las bestias cuando las excitaciones del alcohol avivan la brutalidad de sus instintos sin disciplina. El Perú debe su desgracia a la raza indígena, el indio no es ni puede ser sino una máquina".
\end{abstract}

Las políticas educativas desarrolladas en el Perú hasta la década de 1960 estaban dirigidas a castellanizar a los pueblos originarios, es decir, a la homogenización idiomática. La década del 70 marca el inicio de un proceso de cambios en el país que va a remecer nuestra visión de país, dividido culturalmente.

La tendencia homogenizadora fue quebrada durante el gobierno de Juan Velasco, el cual introdujo un extenso programa de reformas que significaban un nuevo reconocimiento de la composición multiétnica y multicultural del país. Precisamente, la reforma del año 1972 concretó la Política Nacional de Educación Bilingüe (PNEB), entre cuyos lineamientos destacan la necesidad de superar las condiciones de pobreza de los vernáculo hablantes, así como la necesidad de propiciar una interpretación crítica de la realidad social y económica, y de generar una participación espontánea, creadora y consciente en el proceso de cambio estructural, orientado hacia la eliminación de los mecanismos de dependencia y dominación (Vásquez et al. 2009). Ciertamente, la PNEB no incluyó el concepto de "interculturalidad" de manera explícita, pero sus lineamientos evidencian una preocupación por dicha problemática.

La primera política que incorporó el concepto de educación intercultural fue la Política de Educación Bilingüe Intercultural (PEBI) de 1989. Sin embargo, lo hizo desde el enfoque, entonces, vigente el cual limitaba la interculturalidad educativa a la inclusión de conocimientos de la cultura de los vernáculos hablantes y a la gradual incorporación de contenidos procedentes de otras tradiciones 
culturales. Por su parte, la Política de Educación Intercultural y de Educación Bilingüe Intercultural (PEIEB) de 1991 amplió los alcances de este enfoque al concebir la interculturalidad como principio rector de todo el sistema educativo.

\section{GLOBALIZACIÓN E INTERCULTURALIDAD: NUEVAS NECESIDADES EDUCATIVAS.}

La consolidación del proceso de globalización hace posible que nos conozcamos y reconozcamos que el planeta alberga un sinfín de culturas. No existe un país que pueda decir que sólo posee una cultura. En cada país y en cada sociedad nacional coexisten diversas culturas. Esta realidad, que se presenta con mucha fuerza y dinámica socio cultural vertiginosa, afianza la necesidad de repensar las relaciones entre culturas y el término de "Interculturalidad" (que no significa otra cosa que "relación entrte culturas") empieza a ser considerado 'el concepto' idóneo para definir un nuevo tipo de relaciones, que superen la asimetría tradicional y que se sustenten en principios democráticos de tolerancia, respeto y convivencia armónica, generando relaciones de igualdad y equidad.

Interculturalidad, la conceptualizamos como el diálogo permanente entre culturas para enriquecerse mutuamente y aportar al desarrollo de la humanidad. Con la interculturalidad consideramos que se debe garantizar el respeto por la diversidad cultural y fomentar el diálogo cultural para lograr la articulación e integración de prácticas y costumbres, sin atentar contra la identidad cultural de los pueblos nacionales. Vista como conducta, la interculturalidad es la capacidad y habilidad para desenvolverse en contextos de relación de culturas y un saber manejarse entre miembros de diferentes culturas con quienes se interactúa.

De esta manera, la interculturalidad empieza a ser una propuesta de un nuevo modo de vida en todo el mundo, obligándonos a tener que desarrollar nuevas competencias a nivel intra e interpersonal, de valoración interna y social sobre lo que significa vivir en una sociedad altamente diversa culturalmente, por origen, y que se diversifica aún más por las nuevas relaciones que se establecen por la expansión de la llamada globalización, la misma que promueve la movilización de las personas, quienes se trasladan a otras realidades geográficas y socioculturales, pero llevando sus "maletas culturales". Cuando estas personas migran individual o familiarmente, suelen asimilarse a la nueva cultura; pero, cuando lo hacen masivamente, muchas veces bajo circunstancias obligadas (desplazados), el proceso de asimilación se complejiza y se vuelven un grupo más, dentro de la diversidad cultural del país que los recibe.

En el contexto descrito, las personas estamos obligadas a sr interculturales y esto es un proceso de aprendizaje. Se requiere el desarrollo de nuevas actitudes, aptitudes, conductas, comportamientos; por tanto, se presentan dos nuevas nuevas necesidades educativas a nivel de las personas: 
- Una, prepararse para desenvolverse en la diversidad cultural interna del país, de la sociedad de la que se forma parte, para desterrar prejuicios y estereotipos negativos que conllevan a la discriminación de los diferentes grupos étnicos con los que se convive.

- Otra, prepararse para tener una conducta intercultural con las personas que provienen de toda la diversidad cultural del mundo, sin caer en actitudes extremas de xenofobia o de sometimiento, por ideas erradas de superioridad o inferioridad (ésta última por nuestro trauma colonial).

En ambos casos, el proceso de aprendizaje podrá ser natural como parte de la socialización de las personas o planificado, es decir, formalmente y es indispensable contar con docentes formados en interculturalidad.

\section{INTERCULTURALIDAD, EDUCACIÓN INTERCULTURAL Y DOCENTES INTERCULTURALES.}

Los docentes no solo cumplen funciones en las aulas, sino también en toda la comunidad, por lo que ellos son los actores sociales ideales para convertirse en los agentes de la interculturalidad, tanto para los estudiantes iniciales, como para los padres de familia que sin estar directamente en las aulas deben también ser preparados en interculturalidad.

Para este rol, los docentes deben convertirse (léase prepararse) en personas interculturales, por lo que necesitan deconstruir y reconstruir sus competencias con relación a la diversidad cultural. Deben reconocer los prejuicios y estereotipos con respecto a los indígenas andinos y amazónicos, que son la carga cultural que han heredado de la sociedad imaginada como homogénea, para superarlos con los conocimientos certeros y valorativos de esos "otros" culturales, reconocerlos como grupos sociales y culturales. En esa nueva visión es indispensasble que se desarrollen nuevas conductas, habilidades, valores y actitudes como personas y como docentes.

Desde la década de los años 90, antropólogos y sociólogos llamaron la atención sobre la comunidad imaginada del país, como una sociedad que se identifica con lo blanco/criollo que rechaza lo indígena, lo cholo, lo negro, que además se refuerza con prácticas y acciones humillantes a los alumnos rurales o provincianos frente de lo blanco y urbano.

Igualmente, en la educación, a pesar de las políticas educativas de Educación Bilingüe y Educación Bilingüe Intercultural de los años 90, encontramos la misma política de homogeneización que, sin bien no elimina completamente las diferencias culturales las difumina destacando el pasado incaico, promueve una imagen folclórica del indígena andino, tergiversando su identidad bajo la denominación de campesino, 
subliminando características ancestrales sociales y culturales y frente al cual invisibiliza lo amazónico y lo negro o afroperuano (Citarella, 1990; Degregori, 1999).

Aunque la pluriculturalidad peruana está recientemente incorporada como contenido transversal de la educación, aun no hay un tratamiento profundo de la interculturalidad. Todavía, sigue estando relacionada con la educación para los indígenas, como una propuesta de aprendizaje unidireccional desde lo indígena con respecto a la sociedad nacional.

Sin embargo, el hecho de limitar la interculturalidad a los que no manejan el castellano, implica negar las diferencias y profundiza la discriminación (Arenas, 1999, citado en Walsh, 2005). Esta situación debe ser cambiada y es fundamental que los docentes transformen su visión de la interculturalidad para comprender que no se trata solamente del respeto afectivo por los indígenas, a los que en el fondo se subestima, sino que debe ser entendida como el reconocimiento de saberes, experiencias y valores que, potencialmente, pueden contribuir, articuladamente a construir una sociedad realmente democrática, equitativa, sostenible y humana, con diferencias pero sin desigualdad.

La interculturalidad ha operado durante la historia peruana en una forma folclórica, dominante y unidireccional, reconociendo las raíces incas y al mismo tiempo impulsando la asimilación y aculturación de los pueblos indígenas a una identidad nacional homogenizada. Es necesario iniciar un proyecto de interculturalidad de vía múltiple que se base en la convivencia de la diferencia (muchas veces conflictiva), el reconocimiento de la desigualdad y la necesidad de construir sociedades, instituciones y esquemas (legales, educativos, de conocimiento, etc.) distintos (Walsh, 2005).

El Ministerio de Cultura ha venido impulsando la aprobación de normas en materia de pueblos indígenas, población afroperuana, discriminación étnico-racial y ciudadanía intercultural, las cuales han contribuido a fortalecer el marco jurídico de inclusión social de diversos grupos culturales y la aplicación del enfoque intercultural; a pesar de ello resultó necesario contar con una norma nacional que pueda orientar las acciones bajo una visión común que articule e integre la política y gestión en intercultural del país.

En ese sentido, el 28 de octubre de 2016 se aprobó la Política Nacional para la Transversalización del Enfoque Intercultural, con el objetivo de orientar, articular y establecer los mecanismos de acción del Estado para garantizar el ejercicio de los derechos de la población culturalmente diversa del país, especialmente de los pueblos indígenas y la población afroperuana, promoviendo un Estado que reconoce la diversidad cultural, opera con pertinencia cultural, contribuye a la inclusión social, la integración nacional y la eliminación de la discriminación. Tiene como horizonte la construcción de una sociedad intercultural. 
Es en este marco legal que el Ministerio de Educación del Perú, aprobó la política sectorial de Educación Intercultural y Educación Intercultural Bilingüe como principal instrumento orientador para la aplicación de planes, programas y proyectos educativos en esta materia. El Decreto Supremo 006-2016 que aprueba la política señala que es de obligatorio cumplimiento por todas las entidades del sector educación, incluidas las direcciones regionales de educación y las unidades de gestión educativa locales, en el marco de sus competencias. Según la norma, la educación intercultural para todos y todas es la política que orienta el tratamiento pedagógico de la diversidad en todo el sistema educativo, de manera contextualizada, reflexiva y crítica.

Esa norma, busca que cada estudiante construya una identidad desde la afirmación de lo propio y en relación con lo culturalmente distinto, así como el fomento de una convivencia basada en la comunicación, el respeto y el establecimiento de relaciones de complementariedad. "La educación intercultural para todos y todas promueve una ciudadanía que apuesta por un modelo democrático de sociedad, por lograr objetivos comunes de desarrollo como país y, además, enfrenta las prácticas de discriminación, sexismo y racismo" (Declaración de Elena Burga Cabrera, Directora General de Educación Básica Alternativa, Intercultural Bilingüe y de Servicios Educativos en el Ámbito Rural (Digeibira)-2016).

Es importante destacar que la Educación Intercultural, planteada como política y no como acción afirmativa, garantiza que sea un verdadero modelo de educación que implica un proceso de aprendizaje entre las culturas que se relacionan y no una superposición de la cultura dominante sobre la cultura subordinada, la cual tendría que asimilar los valores, prácticas y comportamientos de la dominante.

En consecuencia, el problema de la educación no se asume como un problema exclusivo de los indígenas, sino que atañe a todos los sectores de la sociedad. Con la Educación Intercultural como política se apuesta por el diálogo, el respeto y la convivencia; se atacan no solo las consecuencias de la desigualdad entre culturas, sino sus causas estructurales, articulando justicia cultural con justicia social.

Enfatizamos que los objetivos prioritarios de la educacion intercultural se deben centralizar en:

- Lucha contra los prejuicios étnicos.

- Incorporación del conocimiento del otro.

- Búsqueda de diálogos culturales más equitativos.

- Manejo de un enfoque de equidad de género.

- Revertir condiciones desfavorables de desarrollo para lograr un desarrollo sostenible y humano.

Toda esta propuesta debe desarrollarse bajo la idea rectora de entender la interculturalidad como un proceso y un proyecto social político dirigido a la construcción de sociedades, relaciones y condiciones 
de vidas nuevas y distintas que va mucho más allá del respeto, la tolerancia y el reconocimiento de la diversidad y que apunta a transformar no sólo las condiciones económicas inequitativas sino también a aquellas que tienen que ver con la cosmología de la vida en general, incluyendo los conocimientos y saberes, la memoria ancestral, y la relación con la madre naturaleza y la espiritualidad, entre otras (Walsh, 2008).

\section{A MANERA DE CONCLUSIONES: REQUERIMIENTOS PARA LA FORMACIÓN DEL DOCENTE INTERCULTURAL.}

Revisando las propuestas curriculares de la Universidades que poseen Facultades de Educación, encontramos que, en general, la universidad no forma, en el pregrado, maestros para un país pluricultural y multilingüe como es el Perú, ya que prioriza la enseñanza monolingüe- española y la cultura occidentalizada que es la dominante, sin tener en cuenta la diversidad cultural andina y amazónica. Esta realidad, ha quedado reservada para la formación de maestros bilingües en los Institutos y en algunas Universidades, los mismos que irán a desempeñarse en las escuelas rurales donde el idioma materno no es el castellano. Sin embargo, ahora requerimos un docente intercultural para todo el país, urbano, rural y en los diferentes espacios geográficos, háblese o no otro idioma que no sea el castellano.

Como se planteó en esta comunicación, la realidad peruana, tanto en su estructura interna, como por su inserción en la globalización, presenta un desafío educativo y necesita contar con docentes con nuevas competencias que llamamos interculturales. Este docente requiere contar con una formación no solo en metodologías adecuadas para trabajar con grupos heterogéneos con diferentes niveles de aprendizaje, varios grados, edades distintas, nivel lingüístico diverso, sino sobre todo contextualizar el proceso de enseñanza-aprendizaje a la realidad geográfica, socioeconómica y cultural de cada lugar donde esté y que, cualquiera que éste sea, presentará la pluriculturalidad del país y la presencia de la diversidad cultural internacional.

Un educador intercultural, que forme ciudadanos para la transformación social, superando las asimetrías que se sustentan en prejuicios y estereotipos, mal concebidos por la herencia colonial, debe ser una persona con mucha creatividad, que potencie su capacidad innovadora produciendo sus propias iniciativas.

El nuevo educador que exige la interculturalidad debe considerar que el conocimiento no es objeto sino acción; asimismo, debe ser capaz de potenciar la visualización de nuevos imaginarios de la vida, para lo cual debe valorar e incentivar el protagonismo de los estudiantes, a lo largo de todo el proceso de enseñanza-aprendizaje, remarcando la revaloración de los saberes ancestrales, de sus propias culturas, 
diferentes pero no desiguales y que una verdadera interculturalidad para la decolonización, haga posible que se articulen en igualdad de condiciones, sin dominados y dominados, superiores e inferiores.

Las competencias indispensables que debe orientar la formación del docente, consideramos que son:

- Conocimiento y valoración de la diversidad cultural dentro de la sociedad: respeto por las minorías.

- Compresión de la relación entre culturas en igualdad dentro de las diferencias culturales: la diferencia cultural tiene su propio valor que puede articular los grupos sociales en beneficios equitativos.

- Valoración positiva de la diversidad cultural y acercamiento crítico a los valores culturales diversos: búsqueda de lo que nos puede unir en objetivos comunes y, por tanto, beneficios para todos.

- Respeto y tolerancia a las identidades culturales diversas: saber ser y saber convivir, generando proyectos educativos que involucren a los padres de familia.

- Intercambio de saberes y conocimientos basado en el respeto y la autenticidad: saber aprender y saber hacer, compartiendo experiencias de vida y saberes ancestrales, articulados a ciencia y tecnología.

- Desarrollo de habilidades reflexivas de diálogo y empatía intercultural: desarrollo de actividades educativas que promuevan la solidaridad y el compromiso.

- Apertura a la comunicación intercultural, bajo los principios de nada es inmutable, nada está cerrado, no hay posiciones universales y los conflictos se resuelven.

Finalmente, debemos destacar que consideramos primordial fomentar la formación intercultural del profesorado a fin de que pueda tener herramientas y recursos teórico-prácticos adecuados para desarrollar acciones educativas interculturales tanto al interior de la escuela como en el entorno de la misma, con toda la comunidad educativa. 


\section{REFERENCIAS BIBLIOGRÁFICAS:}

Arguedas, J.M. (2001). Todas las sangres. Lima, PEISA. Gran Biblioteca de Literatura Peruana El Comercio, Tomo 5.

Citarella, L. (1990). "Perú" en F. Chodi (compilador), La educación indígena en América Latina. Tomo II, P.EBI/Abya Yala, Quito.

Cruz Rodríguez, E (2015), "La interculturalidad en las políticas de educación intercultural”. Praxis \& Saber [en linea $]$ (Julio-Diciembre). Disponible http://www.redalyc.org/articulo.oa?id=477247216010

Degregori, C.I. (1999) "Estado nacional e identidades étnicas en Perú y Bolivia”, en K.Koonings y P. Silva (editores), Construcciones étnicas y dinámica sociocultural en América Latina, Abya Yala, Quito.

Degregori, C.I. (2003). Perú: identidad, nación y diversidad cultural. En: Oliart, Patricia (ed.). (2003). Territorio, cultura e historia : materiales para la renovación de la enseñanza sobre la sociedad peruana. Lima: Proeduca-GTZ: IEP Instituto de Estudios Peruanos

Degregori, Carlos Iván

Deustua E., A. (1937). La cultura nacional. -- Lima: [Empresa Ed. de El Callao], 1937

Leiva O, J.J. (2006). "La formación intercultural del docente: reflexiones en el ámbito de la formación inicial y permanente". Revista Comunicación. Volumen 15, año 27, No. 1, Enero-Julio, 2006 (pp. 57-62) Ramírez Villacorta, Yolanda. (2002). Interculturalidad y Educacion. Texto Autoinstructivo. Programa de Licenciatura - UNMSM (anillado)

Tozzini, María Alma et. al. (2015) La interculturalidad en la formación docente: contextos para pensar un recorrido situado. Los casos de El Bolsón y Lago Puelo. En: KIMÜN- Revista interdisciplinaria de formación docente. Año $1 \mathrm{~N}^{\circ} 1$ Septiembre- Diciembre 2015

Tubino, F. (2007). "Las ambivalencias de las acciones afirmativas". VVAA, Educar en ciudadanía intercultural [91-110]. Lima: Universidad de la Frontera y Pontificia Universidad Católica del Perú.

Vásquez, E., et.al. (2009). Niñez indígena y educación intercultural bilingüe en el Perú: Estadísticas recientes, preguntas (i)resueltas y tareas pendientes. Lima: Care Perú, Educa, Save the Children, Tarea y

Unicef. pp. 41-130. Disponible en: http://www.savethechildren.org.pe/wpcontent/uploads/2010/06/ninez-indigena-educacionintercultural-bilingue.pdf

Walsh, C. (2005). La interculturalidad en la Educación. Dirección Nacional de Educación Bilingüe Intercultural. Ministerio de Educación. Lima-Perú.

Walsh, C. (2008). "Interculturalidad, plurinacionalidad y decolonialidad: las Insurgencias políticoepistémicas de refundar el estado”. Tabula Rasa, 9, (julio-diciembre 2008), 131-152.| 